\title{
Announcing the Interdisciplinary College 2009 (IK 2009)
}

\author{
6-13 March, 2009 at Günne, a charming village at Lake Möhne in central Germany
}

\author{
Herbert Jaeger · Thomas Kammer • \\ Gregor Schöner
}

Published online: 10 January 2009

(C) Marta Olivetti Belardinelli and Springer-Verlag 2008

\section{What is the IK?}

The Interdisciplinary College (IK) is an annual 1-week spring school which offers a dense, intense, state-of-the-art course program in neurobiology, neural computation, cognitive science/psychology, artificial intelligence, robotics and philosophy. It is aimed at students, postgraduates and researchers from academia and industry. By combining humanities, science and technology, the IK endeavours to intensify dialogue and connectedness between the various disciplines. Participants come mainly from the European countries, lecturers from all over the world. The course program includes up-to-date introductions to the four main fields of the IK, i.e. Neurobiology, Neuroinformatics, Cognitive Science, and Artificial Intelligence, as well as in-depth courses and discussions of a special focus theme. Additionally, the IK is a unique social event. In the evenings participants may enjoy the very special atmosphere: minds meet, music is played, and friends are made in long evening and night sessions in the welcoming conference site at Lake Möhne.

\section{IK 2009, Focus theme "Rhythm and Timing"}

We live-we breathe, we walk, we speak and think-our being is carried by the flow of time. This is as natural to experience as it is hard to understand. The IK 2009 spins around the scientific riddles of time in life-as it is reflected in neural, cognitive, social and also cultural systems. A wide selection of courses will be elucidating time's enigmas from manifold angles: biological clocks and rhythms; temporal processing in biological and artificial neural networks; timing in motion control, speech, social interactions; oscillation and synchronization in neurons, brains, bodies, and societies; perceiving and generating meter in music and dance; subjective and cognitive experience of time; reasoning about time, planning, acting and decision-making in AI systems; accounts of time in philosophy. A number of methodological courses will provide introductions to scientific methods for grasping time. Miniworkshops from music and the fine arts will keep everyone bodily and sensorially grounded.

About two thirds of the course offering will be directly related to the focus theme. In addition, and in tune with the philosophy of the Interdisciplinary College, there will be up-to-date introductions to the core disciplines of the IK, and specialized courses and hefty evening lectures will highlight recent developments in the sciences of cognition.

We hope that the 2009 edition of the IK will, again, come true as an unforgettable event of learning and inspiration, where-once in a year-disciplinary borders are crossed so freely and vigorously that new levels of understanding are scaled.

An overview of the complete program is available at http://www.ik2009.de. 